\title{
MEMORIAS Y CONOCIMIENTOS INDÍGENAS: EXPERIENCIAS INTERCULTURALES Y EDUCACIÓN EN EL SUDOESTE DE LA PROVINCIA DE BUENOS AIRES
}

\author{
Hernán Perriére* \\ hernanperriere@hotmail.com \\ CONICET, Universidad Nacional del Sur, Argentina
}

Recibido: 11/08/2020 - Aceptado: 6/12/2020

\section{Resumen}

En este artículo presento un análisis sobre los procesos de producción de memorias y conocimientos indígenas en diferentes contextos educativos en el sudoeste de la provincia de Buenos Aires (Argentina). El objetivo es analizar diversas propuestas educativas vinculadas a la interculturalidad donde participaron integrantes de los pueblos indígenas desde un enfoque etnográfico. Para esto, el centro de la investigación son las prácticas desplegadas por una organización indígena urbana de la ciudad de Bahía Blanca y su participación en el Programa de Educación Intercultural, en la Mesa de Trabajo Autogestionada de Educación Intercultural en la provincia de Buenos Aires y en los talleres de alfarería del programa Envión de Bahía Blanca durante los años 2013 y 2018. Esto me permitirá reconstruir una visión amplia sobre los procesos de educación intercultural que exceden a las políticas educativas de gestión estatal y presentar otras instancias de transmisión de memorias y conocimientos en diversos espacios educativos.

Palabras clave: Memorias - Conocimientos - Pueblos indígenas - Educación - Interculturalidad.

\section{INDIGENOUS MEMORIES AND KNOWLEDGE:} INTERCULTURAL EXPERIENCES

\section{AND EDUCATION IN THE SOUTHWEST OF THE PROVINCE OF BUENOS AIRES}

\begin{abstract}
In this article I present an analysis of the processes of indigenous knowledge and memory production in different educational contexts in the southwest of the province of Buenos Aires (Argentina). The objective is to analyze various educational proposals linked to interculturality where members of indigenous peoples participated from an ethnographic approach. For this, the center of the research is the practices deployed by an urban indigenous organization in the city of Bahía Blanca and its participation in the Intercultural Education Program, in the Self-managed Intercultural Education Working Group in the province of Buenos Aires and in the pottery workshops of the Envión of Bahía

\footnotetext{
*Doctor en Antropología (FFyL. UBA). Diplomado Superior en Antropología Social y Política (FLACSO- Argentina). Profesor y Licenciado en Historia (UNS). Becario posdoctoral del CONICET. Se especializa en el campo de la Antropología y Educación. Es asistente de docencia en la materia Prehistoria y Profesor adjunto de Perspectivas Antropológicas de la Educación en el Dpto. Humanidades (UNS).
} 
REVISTA DE LA ESCUELA DE CIENCIAS DE LA EdUCACIÓN, AÑO 17, NRO. 16, VOL. 1, ENERO A JUNIO DE 2021. PÁGINAS 58-67. ISSN 2362-3349 (EN LíNEA). MEMORIAS Y CONOCIMIENTOS INDÍGENAS: EXPERIENCIAS INTERCULTURALES Y EDUCACIÓN EN EL SUDOESTE DE LA PROVINCIA DE BUENOS AIRES. HERNÁN PERRIÉRE.

Blanca program during the years 2013 and 2018. This will allow me to reconstruct a broad vision about the intercultural education processes that exceed the educational policies of state management and present other instances of transmission of memories and knowledge in various educational spaces.

Keywords: Memories - Knowledge - Indigenous peoples - Education - Interculturality.

\section{Introducción}

En la Argentina, con la sanción de la Ley de Educación Nacional №26206 (LEN) en el año 2006, la educación intercultural adquirió relevancia nacional, en particular, con la modalidad de Educación Intercultural Bilingüe (EIB). Esta normativa tuvo su correlato con la sanción de la Ley de Educación de la provincia de Buenos Aires (2007) y sus respectivas referencias a la interculturalidad, un asunto que analizaré más adelante. Sin embargo, es necesario anticipar que la provincia de Buenos Aires no ofrece propuestas específicas de Educación Intercultural Bilingüe en su territorio. Este caso es paradigmático, ya que según los datos censales de 2010 se reconocen como indígenas o descendientes de indígenas 299.311 personas, y es la provincia que cuenta con mayor población que se considera indígena (Hecht; García Palacios; Enriz y Diez, 2015).

Ahora bien, la ausencia de políticas educativas vinculadas a la EIB en la provincia de Buenos Aires, no obstaculiza el debate y la disputa sobre los sentidos de la interculturalidad. Desde este panorama, en este artículo me propongo analizar los diferentes procesos de producción de conocimientos y memoria indígenas en torno a diversas propuestas educativas vinculadas a la interculturalidad, donde participaron integrantes de los pueblos indígenas.

En este sentido, las experiencias que presento intentan enlazar distintos momentos de la investigación etnográfica referidos a prácticas educativas centradas en la interculturalidad que, como explicaré más adelante, exceden a la institución escolar. La intención no es generalizar una dicotomía entre el sistema educativo escolarizado y otras prácticas educativas sobre la interculturalidad, sino mostrar cómo las personas producimos conocimientos y memorias de manera dinámica, según nuestros procesos de identificación étnica, de clase y género. En este sentido, la educación intercultural debería involucrar a todos los sujetos y por lo tanto es debatida en las políticas educativas estatales y también en colectivos y organizaciones civiles en torno al reconocimiento de derechos (Villa; Thisted; Martínez; Diez; 2009).

Para esto, el foco se centra en las prácticas desplegadas por una organización indígena urbana de la ciudad de Bahía Blanca y en su participación en tres instancias educativas desarrolladas durante los años 2013 y 2018: en el Programa de Educación Intercultural, en la Mesa por la Educación Intercultural (ambas de la provincia de Buenos Aires) y en los talleres de alfarería del programa Envión de Bahía Blanca².

El escenario donde se desplegaron estas prácticas es el sudoeste de la provincia de Buenos Aires (Argentina) centralmente en la ciudad de Bahía Blanca y en menor medida en la localidad de Sierra de la Ventana. En dicha ciudad en particular y en el sudoeste de la provincia en general, históricamente la idea de "progreso" (en el sentido civilización-barbarie) se estableció como hegemónica en los discursos oficiales, en la prensa local y, menor medida, en algunos espacios académicos con la finalidad de invisibilizar la presencia indígena en la región. Esta idea se sedimentó con la presencia escenográfica de los espacios militares como son los fuertes y fortines reconstruidos (Nagy, 2013; Pupio; 2013).

Para explicar las formas de producción de conocimientos y memorias indígenas, recupero distintas instancias de investigación etnográfica desarrolladas entre los años 2013 y 2018 en el marco de una investigación de tesis doctoral. Si bien el objetivo de la tesis fue analizar la vinculación entre las escuelas secundarias, los museos y el patrimonio desde una mirada intercultural, la amplitud del trabajo de campo me llevó a compartir distintos espacios educativos en los que la organización indígena participó: centralmente sus charlas en las escuelas y en los encuentros de la Mesa de Trabajo Autogestionada en Educación Intercultural (en adelante, MTAEI).

El trabajo etnográfico que sustenta este artículo incluyó diversas técnicas de recolección de datos: observación participante en los espacios antes mencionados, la realización de entrevistas en profundidad a integrantes de la organización con la finalidad de rescatar las voces indígenas, sus acciones y prácticas, y el análisis de documentos referidos a las leyes de educación nacional y de la provincia de Buenos Aires y al Marco General de la Política Curricular.

Este texto se inscribe dentro de las investigaciones del campo de la Antropología y Educación, campo en el que las discusiones y problemáticas vinculadas a la interculturalidad han tenido un avance importante, sobre todo aquellas orientadas a pensar su relación con los procesos educativos de sectores indígenas y migrantes en la Argentina (Novaro, 2011; Hecht, García Palacios, Enriz y Diez, 2015). Una de las primeras aproximaciones teóricas de este campo, consistió en retomar los estudios que distinguen a la escolaridad de la educación, problematizando así las experiencias educativas dentro y fuera de las escuelas.

Para ampliar esta idea, recupero el concepto de "persona educada" (Levinson y Holland, 1996), que se refiere a una característica común presente en todas las sociedades humanas productoras de cultura, más allá de las formas que adquiera la institución escolar, e incluso también, distante de la

\footnotetext{
${ }^{2}$ Agradezco los comentarios de Ixs evaluadorxs que fueron fundamentales para mejorar este artículo.
} 
educación patrocinada por los grupos dominantes y el Estado. De la misma manera, el concepto de "producción cultural" (Levinson y Holland, 1996) es una construcción teórica que permite describir e interpretar la manera en que la gente se apropia activamente de las condiciones ideológicas y materiales brindadas por la escolarización en sus contextos cotidianos.

En el campo específico de la Antropología y la Educación, la distinción entre la ciencia que se legitima en el sistema educativo por medio del currículum y de otras formas de conocer el mundo, reveló el interés por el estudio de maneras de conocer definidas como "locales", "tradicionales", "intuitivas", "ordinarias" y "practicas" problematizando la manera por las cuales se produce conocimiento. Actualmente, en el campo de la Antropología está en debate la equivalencia de los saberes pretendidamente universales, codificados y academizados de la ciencia y la tecnología y los conocimientos prácticos, locales, implícitos y corporizados del sentido común (Padawer; Canciani; Greco; Rodríguez Celin; Soto, 2017).

En este artículo, en primer lugar, presento un breve diálogo entre una de las integrantes de la organización indígena que participó del Programa de Educación Intercultural impulsado desde la Dirección General de Cultura y Educación (DGCyE) de la provincia de Buenos Aires, con una docente que asistió a la charla que la organización dio en una escuela secundaria. Para contextualizar este intercambio, retomo la vigencia de los marcos normativos sobre el tratamiento de la interculturalidad en el sistema educativo, con especial énfasis en el artículo 44 de la Ley de Educación de la provincia de Buenos Aires. En segundo lugar, describiré dos experiencias vivenciadas junto a integrantes de la MTAEI cuya demanda principal es requerirle al Estado el cumplimiento del artículo 44 de la legislación provincial. Estas, son experiencias impulsadas desde diversos colectivos indígenas y de otros sectores que apoyan sus demandas. Además, la MTAEI es un espacio para encontrarse, debatir, rememorar y producir conocimientos anclados en su cosmovisión como pueblos indígenas. Por último, haré referencia a la transmisión de conocimientos y memorias indígenas que una integrante de la organización realizó como tallerista de alfarería mapuche en el programa Envión de Bahía Blanca. En su relato, ella narra cómo trasmite sus conocimientos de mujer alfarera a adolescentes y jóvenes en contextos de vulnerabilidad social.

\section{Políticas educativas e interculturalidad}

Como he mencionado, en el año 2006 la EIB surge como una política educativa nacional destinada centralmente a los pueblos indígenas. La legislación concerniente a EIB en el país toma como base de sus declaraciones al Artículo 75, inciso 17, de la Constitución Nacional (reformada en 1994). ${ }^{3}$

En la Ley de Educación de la provincia de Buenos Aires N. o 13688/07, el capítulo XIII se refiere a la educación intercultural. En el artículo N. ํ 44 menciona que los objetivos y funciones de la Educación Intercultural son:

1) aportar propuestas curriculares para una perspectiva intercultural democrática impulsando relaciones igualitarias entre personas y grupos (...) en vistas a la construcción de una sociedad inclusiva, 2) formular proyectos de mejoramiento y fortalecimiento de las instituciones y programas que (...) integren las particularidades y diversidades de la provincia, sus habitantes y culturas, propiciando el respeto a la diversidad cultural (...); 5) impulsar la construcción de orientaciones pedagógicas y curriculares interculturales, así como la inclusión de la perspectiva intercultural en la formación y actualización docente para todos los niveles del sistema educativo, 6) contribuir a asegurar el derecho de los Pueblos Originarios y comunidades migrantes a recibir una educación intercultural y/o bilingüe que ayude a preservar, fortalecer y recrear sus pautas culturales, sus lenguas, sus cosmovisiones, sus tradiciones e identidades étnicas, 7) incentivar la formación de espacios de investigación (o centros de estudio) en Educación Intercultural, con la participación de las Universidades nacionales y provinciales, los Centros de Investigación Educativa, los Institutos de Formación Docente y otros organismos y organizaciones interesadas para el diseño de prescripciones curriculares, materiales educativos pertinentes e instrumentos de gestión pedagógica". ${ }^{4}$

\footnotetext{
${ }^{3}$ La EIB retoma estas normativas: Resolución N. o 107 del Consejo Federal de Cultura y Educación [CFCE] (1999); Resolución N. 549 del Ministerio de Educación Ciencia y Tecnología de la República Argentina (2004); Documento del Programa Nacional de Educación Intercultural Bilingüe de la Dirección Nacional de Programas Compensatorios Subsecretaría de Equidad y Calidad Educativa del Ministerio de Educación, Ciencia y Tecnología de la República Argentina (2004); Ley de Educación Nacional N. 026206 (2006); Documento para la Modalidad de Educación Intercultural Bilingüe en el Sistema Educativo Nacional del Consejo Federal de Educación (2010) y Resolución N. ㅇ 105 del Consejo Federal de Educación (2010).

${ }^{4}$ Esta idea se retoma en los diseños curriculares comunes. En la Resolución N. ㅇ 3655/07, referida al Marco General de la Política Curricular de la provincia de Buenos Aires, se señala como propósito formar sujetos que reconozcan y valoren la diversidad, desde esta perspectiva, la interculturalidad se incluye en los diseños curriculares como enfoque, estrategia y contenido. Como enfoque, porque supone un posicionamiento pedagógico desde la heterogeneidad cultural. Como estrategia, al identificar diferentes escenarios escolares, sujetos y modos de conocer y diseñar intervenciones que los pongan en diálogo. Por último, como contenido, al incorporar temas, nociones y saberes relacionados a las identidades culturales en los cuales los alumnos y alumnas se reconocen. Según esta resolución, se propone una intervención dirigida a orientar y favorecer la interrelación entre culturas desde una perspectiva intercultural que valore la interacción y comunicación recíproca y comprenda las diversidades desde una perspectiva de derechos.
} 
La explicitación de la legislación permite señalar que el sistema educativo provincial valora la perspectiva intercultural y los derechos de los pueblos indígenas, a la vez que incentiva la renovación de los espacios de investigación de educación intercultural en la formación superior. Sin embargo, el análisis de las prácticas educativas a "escala cotidiana" (Rockwell, 2009) desarrolladas por las integrantes de la organización (generalmente charlas) en las escuelas, permitió tensionar la implementación de estas políticas educativas a la que la legislación hace referencia.

En varias oportunidades $(2014,2016$ y 2018) pude registrar distintas charlas que las integrantes de la organización indígena desarrollaron en las escuelas de diversos niveles educativos. A los fines de este artículo, solo haré referencia a una de ellas, porque permite poner en discusión la aplicación concreta de las políticas educativas interculturales. En este sentido, seleccioné un fragmento de un diálogo entre docentes de la escuela visitada y una integrante de la organización indígena que participó del Programa de Educación Intercultural de la provincia de Buenos Aires como referente de la organización y además por ser maestra de educación primaria:

Docente (D): - En la última reforma educativa, se reconoce a ser educado en su lengua materna digamos... en la del 2006, cuando se hizo la última reforma, no se implementó en todos lados, pero digamos las leyes te amparaban (...) se hablaba de bilingüismo y ya no se pensaba en el inglés como siempre, sino en el derecho a su lengua (se refiere a la de los pueblos indígenas) Referente indígena (RI): - Costó mucho la implementación de la ley. Una cosa han sido las normas y otra cosa es que eso se pueda llevar a cabo en las escuelas. Incluso todo lo que había de educación intercultural se dio marcha atrás, acá en la provincia. Entonces, ya no hay. La gente que quiere trabajar en proyectos que tienen que ver con pueblos originarios, lo tiene que hacer como docente individual...

D: - ¿La comisión no está más? (en referencia al Programa de Educación Intercultural)

RI: - No, no está, yo era referente de educación intercultural y fue menos de un año que estuve trabajando, se preveía un cambio institucional con los referentes trabajando de veinte a treinta años... te imaginás... y después de medio año nadie más siguió trabajando... Lo hacen las docentes de manera particular. No como política educativa.

D 2: - Cuando vos estabas... ¿el programa se dio de baja?

Rl: - A todas nos dieron de baja, como que teníamos que volver al aula, cuando éramos referentes de educación intercultural. Y, en un momento se nos volvió a llamar, pero con la idea de que teníamos que anotarnos como monotributistas, y habíamos acordado no aceptar (...) Somos docentes, al igual que el resto y teníamos que hacer una función como ir a diferentes lugares y bueno, tienen que poner presupuesto e informar bien y tener una buena política de educación intercultural. Si no es como que parece que hacemos pero no hacemos nada. No aceptamos, se consensuó no continuar (Registro de charla de organización indígena, Escuela Secundaria, 17 de mayo 2018).

Este diálogo permite describir la situación de "desarticulación" que estaba atravesando el Programa de Educación Intercultural en la provincia de Buenos Aires en el año 2018 y a la vez marca las dificultades de su implementación. Una de las docentes que participó de la charla destacó el reconocimiento del Estado hacia las lenguas indígenas como un derecho de los pueblos indígenas frente a otros idiomas como el inglés. Su comentario interpeló a la referente de la organización indígena para intercambiar su experiencia personal, detallando lo costoso de la implementación de la legislación. Destacó la contradicción entre lo que expresa la normativa, lo que sucede en las escuela y su trayectoria personal como referente indígena y maestra en el Programa de Educación Intercultural. ${ }^{5}$. De esta manera menciona que ante la ausencia de una política educativa estatal, los decentes que quieran abordar la temática de los pueblos originarios de deben hacer por su cuenta.

En charlas informales con ella, me expresó que su trabajo en el Programa consistió en viajar por las ciudades y localidades del sudoeste bonaerense para realizar reuniones con otros referentes indígenas/maestros y con las autoridades distritales (inspectora/es) con la finalidad de implementar proyectos de educación intercultural. Durante los años 2015 y 2016 las referentes indígenas que integraban el Programa representaban a las ciudades de La Matanza, Olavarría, Junín, Carmen de Patagones, Partido de la Costa y Bahía Blanca. En el año 2016, disminuyeron a la mitad, sólo había referentes de La Matanza, Junín y Carmen de Patagones, no obstante, el proyecto no continuó por una decisión política, desarticulándose el Programa en su totalidad en el año 2017 (Lucas, 2018).

\footnotetext{
${ }^{5}$ Desde la sanción de la LEN (2006), en la provincia de Buenos Aires se estableció la Modalidad de EIB solamente durante el año 2007, bajo la Dirección de Educación Intercultural dependiente de la Dirección General de Escuelas y Educación (ver: Diez, Thisted y Martínez, 2015). Trabajo coordinado entre docentes e investigadoras de Ciencias de la Educación y de Antropología de la Universidad de Buenos Aires y de la Universidad Nacional de La Plata. Posteriormente entre los años 2008 y 2015, se implementa como Programa bajo la administración de distintas Direcciones. En el año 2015 se nombran como referentes a seis docentes indígenas, en el año 2016 se designa a la mitad y en el año 2017 se desarticula el Programa, quedando sólo experiencias regionales o locales (Lucas, 2018).
} 
Sería tema de otro texto analizar las variables entre los idiomas predominantes y las lenguas indígenas subalternas y su articulación con el sistema escolar, pero sí es necesario mencionar brevemente que hay un consenso generalizado en las investigaciones referidas a la interculturalidad de que la incorporación de las lenguas y otros saberes indígenas no modifica la estructura de poder de la escuela tradicional (Diez, Hecht, Novaro y Padawer, 2011). Además, si bien la aplicación de las políticas educativas interculturales es una demanda de los colectivos indígenas, este modelo de la EIB, que promueve la enseñanza de la lengua indígena en la escuela pública deja, con frecuencia, inalterados los modos de organización de tiempos, espacios, relaciones de poder, contenidos y prácticas de la escuela moderna, subordinando la permanencia e integración escolar de niños, adolescentes y jóvenes a los parámetros hegemónicos (Thisted, 2014).

Según lo relatado por la referente indígena, puedo argumentar que la decisión de abandonar el programa definitivamente tuvo que ver con la política extendida durante la presidencia de Mauricio Macri (2015-2019) de convertir a los docentes indígenas del programa en monotributistas, lo cual significaba aportarle al Estado una suma de dinero a la par de aumentar su trabajo como referentes, además de su tarea cotidiana como maestra. Para ella, esto significó una muestra de que la política educativa intercultural requiere de un buen presupuesto y claridad en el hacer.

En relación a los procesos de memorias, Halbwachs (2004) introdujo la idea de "trayectoria", definiéndola como las formas en que las personas reconstruimos nuestras propias autobiografías, que no son lineales, sino que se irán modificando según los lugares que ocupamos y las relaciones que establecemos en los distintos contextos sociales (Ramos, 2011, p. 133). En su trayectoria y en particular en el diálogo mencionado, la referente de la organización indígena hiló sus vivencias personales desde su práctica concreta en un programa de educación intercultural estatal a la que accedió por su rol como maestra.

Esta experiencia permite reflexionar al menos dos cuestiones: una, es que el Estado no garantiza la aplicación de una legislación en la provincia de Buenos Aires, lo cual quedó de manifiesto en la falta de ministerios, secretarías, presupuestos, encuentros con las comunidades indígenas para diseñar los currículums y las capacitaciones docentes. La otra, si es posible que la normativa de educación intercultural por sí sola pueda cambia las profundas desigualdades y la vulnerabilidad de derechos que vivencian los pueblos y comunidades indígenas en la provincia.

En la provincia de Buenos Aires, la legislación educativa contempla, como he mencionado, diversas instancias para el desarrollo de políticas interculturales; sin embargo, las y los referentes/docentes de los pueblos indígenas fueron incorporados al programa en el año 2015, ocho años después de la sanción de la Ley de Educación provincial. Durante estos años, desde el 2012, algunas organizaciones y referentes indígenas impulsaron la MATEI para discutir la implementación de artículo N. ํ 44.

\section{Mesa por la Educación Intercultural de la provincia de Buenos Aires}

La MTAEI de la provincia de Buenos Aires es una mesa itinerante que se reúne desde el año 2012 en distintas ciudades y localidades de la provincia. Esta mesa está integrada por representantes de pueblos, comunidades y organizaciones indígenas (mayormente mapuche) junto a otras instituciones que promueven espacios de debates sobre la educación intercultural. La integran representantes indígenas de Junín, Los Toldos, Olavarría, Bolívar, Tigre, San Pedro, La Plata, Malvinas Argentinas, Florencio Varela, Mar de Ajó, Carmen de Patagones, Rauch y Bahía Blanca (Rosso, 2018). ${ }^{6}$ Entre los objetivos específicos de la Mesa se expresa la aplicación del artículo N. 44 de la ley de educación de la provincia de Buenos Aires presentado en el apartado anterior. Asimismo, esos encuentros itinerantes, expresaron la necesidad de definir a la educación intercultural desde la propia mirada indígena. Estos encuentros son espacios de reflexión, debate y de resoluciones, pero en ellos también se incluyen "ceremonias ancestrales", en algunos casos "con autoridades espirituales originarias", en algunas de ellas he participado, recuerdo particularmente la de Sierra de la Ventana en mayo del 2014. Además, en estos encuentros se despliegan diversas acciones: presentaciones de libros, de trabajos universitarios sobre la interculturalidad, eventos musicales, se abordaron junto a abogadas problemáticas vinculadas a los Derechos Humanos, entre otras.

Entre los años 2012 y 2018 se realizaron 17 mesas de trabajo, tres de ellas en el sudoeste bonaerense: dos en Bahía Blanca (cuarta y sexta durante el año 2013) impulsadas por integrantes de la organización indígena referenciada en este artículo y la novena mesa en el año 2014, en la localidad de Sierra de la Ventana. ${ }^{7}$ A estos tres encuentros de la MATEI asistí acompañando a la organización, además también participé de un encuentro en la ciudad de Olavarría pero excede el escenario de estudio de este artículo. En este sentido, me interesa subrayar acá y, a partir de mi experiencia, dos ejes que se abordaron en la mesa de trabajo: uno de ellos consistió en la elaboración de la definición de educación intercultural y

\footnotetext{
${ }^{6}$ Además participaron otras instituciones como la Defensoría Oficial (Departamento Judicial de Azul), la Comisión Provincial por la Memoria (Programa Pueblos Originarios y Migrantes) y referentes de la Secretaria ejecutiva del Consejo de Asuntos Indígenas de la provincia de Buenos Aires. También integraron la mesa estudiantes, docentes e investigadores universitarios: representantes Facultad de Ciencias Sociales de la Universidad Nacional del Centro (UNiCen), del Departamento de Humanidades de la Universidad Nacional del Sur (UNS), del Ciclo Reencuentros con Pueblos Originarios de la Universidad de General Sarmiento (UNGS). Además integraron la mesa el espacio educativo Arbolito de la ciudad de Rauch e integrantes de la Asociación Cultural "Bahía Che" y de FM de la calle de la ciudad de Bahía Blanca.

${ }^{7}$ Otras se han realizado en Olavarría, Junín, Bolívar, Los Toldos, Lobos, Laguna Sierra de los Padres y Tandil.
} 
el otro, una actividad de mapeo territorial (cartografía social) impulsada por la geógrafa Inés Rosso y los y las organizadores de la mesa en Sierra de la Ventana.

Respecto al primero, la sexta mesa de trabajo se realizó en la ciudad de Bahía Blanca y participaron además de los anfitriones bahienses, representantes indígenas de Olavarría, Los Toldos, Rauch, Lobos y de Carmen de Patagones. En consonancia con los objetivos de este artículo, describiré brevemente distintas discusiones donde a mi entender se articularon procesos de memorias, producción de conocimientos y educación, que construiré y resumiré brevemente retomado las notas de campo. Es necesario aclarar que estos encuentros sólo se registraron con notas de campo, por lo tanto, son parciales y los fragmentos citados a continuación no corresponden específicamente a integrantes de la organización indígena de Bahía Blanca:

"La escuela justifica el genocidio" (participante indígena). Durante todo el encuentro, se fueron expresando intervenciones que discutieron el lugar del Estado y de la escuela marcando una continuidad histórica que se articuló con el genocidio de los pueblos originarios. En algunas de ellas se argumentaba que el Estado siempre desarrolló una perspectiva que mantuvo (y mantiene) la idea de progreso y que esto fue una de las causas del genocidio indígena que la escuela justifica. En este sentido, la MTAEI definió de forma explícita que hubo un genocidio indígena (en referencia a la llamada Conquista al Desierto y otras) y que eso repercutió en la conformación del sistema educativo hasta la actualidad (Notas de campo, Bahía Blanca, 24 de agosto de 2013).

"Somos sujetos históricos. Tenemos que empoderarnos de la historia" (participante indígena). Como consecuencia de lo mencionado en el punto anterior, buena parte del encuentro también discutió la necesidad de construir una "nueva educación" para cambiar la historia, rompiendo los estereotipos sobre los pueblos indígenas, para construir la historia desde otro lugar y en un diálogo permanente entre las comunidades, sus saberes y el Estado (Notas de campo, Bahía Blanca, 24 de agosto de 2013).

En estas referencias, se desplegaron procesos de memorias articulados históricamente con el genocidio indígena y el rol de Estado y la escuela para su justificación. Además, adquirió relevancia "lo colectivo" como forma de apropiarse de la historia, a la vez que se debatió sobre la necesidad de cambiar la historia por medio de la educación. Ambos fragmentos presentan un hilo común que construye memorias sobre el pasado.

Estos procesos pueden analizarse desde el campo de los estudios sobre la memoria y del concepto de "memoria colectiva" desarrollado por el sociólogo Maurice Halbwachs a fines de la década de 1930. Él se refería a los procesos de recuerdo y olvido de grupos o colectividades, advirtiendo que cuando rememoramos algún acontecimiento no lo hacemos solos, sino con los recuerdos de los demás, recuerdos que quedan enlazados y que se refuerzan en conmemoraciones y celebraciones compartidas. Todo lo antedicho, llevó a ubicar a los procesos escolarizados como centrales en la transmisión hegemónicas de memorias oficializadas por el Estado. Por esto se discutió la necesidad de una "nueva educación" donde los saberes indígenas sean relevantes y en diálogo con el Estado. En este sentido, la MTAEI definió como Educación Intercultural a: "la relación igualitaria, simétrica y circular, basada en la aceptación de las diferencias y en la riqueza de la diversidad. En pos de los derechos de los pueblos originarios, a una educación general que respete su cosmovisión, idioma, arte y territorio" (Notas de campo, Bahía Blanca, 24 de agosto de 2013).

Como he mencionado, en este artículo considero a la interculturalidad como campo de disputa de sentidos y prácticas en los cuales se entrelazan cuestiones históricas referidas a las clases sociales, la desigualdad, el ejercicio del poder y la vulneración de derechos indígenas. La definición colectiva de la MTAEI disputa sentidos sobre la interculturalidad porque pone en juego los proyectos de los pueblos indígenas para vivir bajo sus propios sistemas sociales y culturales (Diaz y Rodríguez de Anca, 2014, pp. 174-175).

En el 9 Encuentro que se realizó el 24 de mayo de 2014 en Sierra de la Ventana se desarrolló un taller de cartografía social participativa sobre la presencia indígena en la provincia de Buenos Aires (Rosso, 2018). ${ }^{8}$ El trabajo se realizó en cuatro grupos y como resultado se elaboraron cuatro mapas. Los materiales con los cuales trabajar fueron aportados por la tallerista: cuatro grandes mapas físicos contorneados de la provincia de Buenos Aires dispuestos a ser intervenidos con distintas figuras (iconos) para pegar, con fibras y lapiceras de colores. Esta metodología de trabajo tuvo como finalidad "la necesidad de recuperar conocimientos populares que de otra manera no podían construirse (...) y el sentir que sus saberes son valorados y sirven a una lucha colectiva" (Rosso, 2018 p. 70).

Lo interesante de la propuesta fue que las y los participantes realizaron un mapeo que contenía las experiencias, trayectorias y memorias desde sus propios conocimientos como pueblos y comunidades indígenas. Algunos interrogantes presentados por la coordinadora hicieron referencia a los lugares donde habitaron y habitan las comunidades indígenas, a la denominación de las identidades de los pueblos

\footnotetext{
8 Según Rosso, "la cartografía social se propone como un medio para potenciar determinados modos de pensar y construir el territorio y se presenta como fortalecedora de espacios de resistencia, emergiendo como valiosa instancia de construcción colectiva de conocimientos" (Rosso, 2018, p. 56).
} 
indígenas en la actualidad, como también a los grupos que promueven la recuperación de la identidad indígena. Otras cuestiones se refirieron a los reclamos y conflictos históricos y actuales, y a los movimientos migratorios en el territorio provincial y los "idiomas originarios".

Estos mapeos pusieron y ponen en tensión los procesos hegemónicos de denominación, demarcación y territorialidad impuestos por el Estado nacional en el sudoeste bonaerense y en la actual provincia de Buenos Aires. Además, los mapeos superaron estos interrogantes iniciales explicitando otros conocimientos que los pueblos indígenas despliegan en la actualidad: las ceremonias, el Wizun (alfarería), el Witral (telar), la platería artesanal y el canto mapuche.

Respecto a la educación, un Lonco (autoridad indígena) de una comunidad expresó durante el mapeo: "Nuestra educación viene de nuestros abuelos, no hay abecedario mapuche, el abecedario es prestado de los blancos. No aprendimos de los libros, nuestro conocimiento no se aprende por libros, nuestros ancianos o autoridades tradicionales lo fueron transmitiendo en forma oral" (Notas de campo, Sierra de la Ventana, 24 de mayo 2014).

Es interesante esta afirmación del referente indígena porque describe los procesos de aprendizaje que se transmiten de manera intergeneracional en los cuales la oralidad constituye un elemento central. También permite reflexionar sobre la escritura y el uso del abecedario que le asigna la categoría de "préstamo" cultural de la población blanca. El relato sitúa sobre la agenda una problemática muy importante para pensar la interculturalidad que excede el bilingüismo o la "cuestión" de las lenguas, para instalar otras formas de enseñanza-aprendizaje donde los ancianos son depositarios y protectores del conocimiento. Esta problemática que excede a este artículo, es desde mi mirada, uno de los puntos nodales desde donde pensar la interculturalidad como diálogo de saberes, considerando las jerarquías, las situaciones de poder y las herramientas impuestas desde el Estado.

Recuperar la experiencia de la MTAEI, permite reflexionar sobre los procesos de memoria y conocimientos que los pueblos originarios despliegan en espacios comunitarios, en los que la palabra circula horizontalmente y donde los procesos socioculturales remiten a situaciones conflictivas. La antropóloga Elena Achili (2009) abordó estas experiencias en la comunidad toba de la ciudad de Rosario como procesos de "construcción comunitaria". La experiencia de vida urbana de los grupos indígenas supone complejos procesos en los que se entrecruzan nuevas condiciones de vida con otros procesos socioculturales que remiten conflictivamente a las configuraciones de un "nosotros" en torno a ciertas "identidades políticas" desde las cuales estos pueblos indígenas demandan educación (Achilli, 2009, p. 267).

En la escala cotidiana, los procesos de construcción de memorias y de producción de conocimientos se expresan en los integrantes de la organización indígena en distintas prácticas, algunas educativas y otras más políticas. Es aquí donde las trayectorias personales se hacen colectivas y de militancia cotidiana como la que desenvuelve una integrante de la organización en el Envión.

\section{Conocimientos y memoria mapuche en el programa Envión}

El Programa de Responsabilidad Social Compartida Envión es una política social y educativa destinada a adolescentes y jóvenes (de 12 a 21 años) que se encuentran en situación de vulnerabilidad social y fue diseñado por el Ministerio de Desarrollo Social de la provincia de Buenos Aires (ahora llamado Desarrollo de la Comunidad). En la ciudad de Bahía Blanca hay seis sedes del programa distribuidas en distintos barrios donde asisten alrededor de 1000 chicos y chicas.

Ahora bien, es importante aclarar que a diferencia del Programa de Educación Intercultural y de la MTAEI de la provincia de Buenos Aires, el programa Envión es un espacio educativo, pero no se define desde las políticas públicas de interculturalidad, aunque las dinámicas de los talleres que desarrolla puedan realizarlas. En esta parte, la mirada hacia los procesos de interculturalidad, se focaliza tienen en el rol de una integrante de la organización indígena en el programa.

A lo largo del trabajo de campo, como he comentado, entrevisté a distintas integrantes de la organización indígena a las que acompañé en las charlas escolares y en la MATEI. En este apartado me propongo dar cuenta de los procesos de transmisión de conocimientos que una de ellas desarrolló en el programa Envión. En el momento de la entrevista en el año 2018, ella se desempeñaba como tallerista de alfarería desde hacía más de 10 años, su sueldo lo pagaba la Municipalidad de Bahía Blanca, un tiempo después dejó de trabajar a causa de un proceso de recorte salarial y de inestabilidad laboral para las y los trabajadores del programa. En la entrevista ella comentó que el municipio les exigía que se convirtieran en monotributistas, lo cual requería otros gastos que muchos trabajadores no podían afrontar, al igual que la referente que integró el Programa de Educación Intercultural.

Según relató, ella se sentía orgullosa de trasmitir los conocimientos del trabajo con la arcilla, que aprendió por transmisión cultural familiar, a las y los niños/jóvenes que asisten a Envión. Además de un trabajo, ella sentía que lo que hacía cumplía también un objetivo social, más allá de la enseñanza de la alfarería, tenía que ver con su identidad, con hacer de su trayectoria de vida un aprendizaje que podía transmitir a los demás desde su reconocimiento étnico y de género. Sin embargo, en el siguiente fragmento de la entrevista me detengo en su actividad como tallerista, porque para ella el enseñar a modelar la arcilla era al mismo tiempo una excusa, una vía para trasmitir lo que definía como "la cosmovisión del pueblo mapuche":

E (entrevistador): - ¿Qué haces en esos talleres? 
Tallerista Indígena (TI): - Yo doy taller de alfarería mapuche... Ese es el rol que yo cumplo en el taller de los Enviones. Y además de dar el taller de alfarería mapuche, los chicos aprenden a hacer vasijas, utilitarios, adornos, pero también tienen el plus de que comparto con ellos que me reconozco mujer mapuche. Comparto con ellos algo de lo que es el mapuzungún, que es el habla de la tierra, de la lengua materna, y este fuerte arraigo que tenemos los pueblos originarios con la madre naturaleza. Entonces el hecho de cuidar el medioambiente, de pedir permiso cuando hacemos la extracción de arcilla (...) Yo comparto con los chicos un pequeño willipún, que es una ceremonia en la cual se le pide permiso a la madre tierra para la extracción. Algo que les llama mucho la atención, porque estamos acostumbrados a tomar sin pedir permiso...lo que va totalmente en contra de la cosmovisión de los pueblos originarios (...) Yo soy la cuarta generación de alfareros en la familia y bueno, también ellos pedían permiso a la ñuke mapu, a la madre tierra. (Entrevista a integrante de organización indígena, Bahía Blanca, 4 de mayo de 2018).

El relato de la mujer indígena puede leerse como una "traducción fundada en habilidades" (Padawer, 2013) en tanto permite verbalizar los conocimientos construidos en comunidad, en familia, donde se ponen en juego la relación con la naturaleza y las prácticas cotidianas del hacer aprendiendo. En este sentido, en su narración menciona a la lengua mapuzungún que aprendió de su madre y el fuerte vínculo del pueblo mapuce con la naturaleza. En este proceso de transmisión de conocimientos se despliegan la ceremonia de permiso a la "madre tierra" y el cuidado del mediomabiente.

Otra noción que resulta útil para considerar los conocimientos y prácticas cotidianas que se entraman al recordar es la de "memorias-conocimientos". Tim Ingold (2011) recurre a este concepto para referirse a la memoria como un conocimiento específico, pero que a su vez es un resumen del conocimiento integrado a lo largo de diversas trayectorias, de modo que se trata de analizar cómo los caminantes transitan los senderos ya recorridos por otros. En este sentido, conocer algo o alguien es conocer su historia y ser capaz de juntar aquella historia con la propia, siendo en este entramado donde las "memoriasconocimientos" son generadas. El conocimiento emerge en la vida a través de un proceso activo de recordar y no como una transmisión pasiva del legado de los ancestros, conocer es ser capaz de volver a contar historias del mundo y volver a tejer memorias por donde otros ya pasaron y otros seguirán (Ramos, 2016, p. 55).

La circulación del conocimiento de la organización mapuche excedía los talleres municipales y las presentaciones escolares. En Bahía Blanca se constituyeron como referentes de la visibilidad de la problemática indígena, en el rescate de la memoria, en las demandas militantes por los derechos de los pueblos originarios y otras instancias políticas donde la memoria cobra sentido, por ejemplo, en las marchas de aniversario del último golpe militar, las acciones por los asesinatos de Santiago Maldonado, por Rafael Nahuel, entre otras.

En el campo de los estudios sobre la memoria, historiadores como Eric Hobsbawn y Terence Ranger (2002) propusieron que las sociedades se apropian del pasado para buscar respuestas en el presente, la memoria construye el pasado integrando los textos históricos y los relatos orales en el presente. Para la socióloga Elizabeth Jelin (2018), es el sentido del pasado el que está sujeto a interpretaciones y por esto constituye un escenario de confrontaciones frente a otras interpretaciones, a menudo contra olvidos y silencios. En este sentido, las prácticas de memoria se construyen como "una militancia" sobre el pasado que se expresa en la esfera pública. Las acciones que realiza la organización mapuche trascienden de esa forma lo comunitario en un sentido étnico restringido: su memoria colectiva se presenta como una identidad en permanente construcción, participando en distintas disputas políticas y de difusión cultural como parte del reconocimiento social.

\section{Palabras finales}

A lo largo de este trabajo me propuse dar cuenta de los procesos por los cuales integrantes de organizaciones indígenas producen memorias y conocimientos en distintos espacios educativos de la provincia de Buenos Aires. Señalé que si bien la legislación sobre la EIB es amplia en la provincia, no hay organismos destinados a su implementación, a pesar de la gran cantidad de población indígena que la habita.

Las tres experiencias analizadas permitieron argumentar que pese a esto, los espacios educativos vinculados a la interculturalidad se extendieron de diversas maneras en sudoeste de la provincia de Buenos Aires. De esta manera, mi perspectiva de análisis basada en la investigación etnográfica de las prácticas sociales a escala local y "cotidiana", permitió presentar una visión ampliada sobre la interculturalidad en los procesos educativos en los cuales se entraman conocimientos y memorias que se producen culturalmente más allá de la política educativa escolar expresada en la LEN en todo el territorio nacional. Reconociendo la importancia de las demandas de los colectivos indígenas por la EIB, y sin desconocer las prácticas genocidas del Estado argentino hacia los pueblos indígenas, destaqué que hay importantes procesos educativos donde la interculturalidad continúa siendo tema de debate.

En los relatos sobre el conocimiento en torno al pasado aparecen distintos elementos constitutivos de la memoria. Los "actos del recuerdo" se reactivan en los encuentros de la MTAEI, a la vez que se articulan con otras demandas educativas y políticas. Las "trayectorias" personales relatan un abanico de experiencias que incluyen balances sobre la participación en programas de gestión estatal hasta la enseñanza de la 
REVISTA DE LA ESCUELA DE CIENCIAS DE LA EdUCACIÓN, AÑO 17, NRO. 16, VOL. 1, ENERO A JUNIO DE 2021. PÁGINAS 58-67. ISSN 2362-3349 (EN LíNEA). MEMORIAS Y CONOCIMIENTOS INDÍGENAS: EXPERIENCIAS INTERCULTURALES Y EDUCACIÓN EN EL SUDOESTE DE LA PROVINCIA DE BUENOS AIRES. HERNÁN PERRIÉRE.

alfarería mapuche en talleres municipales. Las "memorias colectivas" que narran las referentes indígenas, entrelazan vivencias personales con demandas de derechos. Al recordar se entraman "memoriasconocimientos" de distintas trayectorias que no se transmiten de manera pasiva ancestralmente, sino que se tejen en el "qué hacer" cotidiano, y se vuelven en nuevas formas de memorias y nuevos conocimientos sobre el tejer en el telar, modelar la arcilla, narrar en mapuce, en las ceremonias, por ejemplo.

Como he mencionado, para poder avanzar en la implementación de la EIB es importante poder dialogar con las experiencias concretas de memorias y producción de conocimientos indígenas que son diferentes a las escolares. Por esto es importante escuchar la voz de los pueblos indígenas para reformular los contenidos del currículo, la capacitación de docentes, la creación de material didáctico tal como lo plantea el artículo N. ․ 44 de la ley de educación provincial.

Por último, interpelo a preguntarnos si es posible plasmar curricularmente estos conocimientos prácticos indígenas ligados al hacer que se recuperan de forma oral "traduciendo las experiencias fundadas en habilidades" a una estructura rígida y jerárquica como es el sistema educativo argentino. Si es posible que las comunidades indígenas ejerciten el derecho a su propia educación, según sus propios conocimientos, estableciendo relaciones interculturales desde sus propias concepciones. Y para finalizar, reflexionar sobre los entramados de la educación con otros procesos que debemos atender como la discriminación, el racismo, el despojo de las tierras, los desmontes, los desalojos, las situaciones de pobreza, que viven los pueblos originarios en la actualidad.

\section{Referencias bibliográficas}

Achilli, E. L. (2009). Escuela. Familia y desigualdad social: una antropología en tiempos neoliberales. Rosario: Laborde Libros.

Diaz R. y Rodríguez de Anca, A. (2014). Activismo intercultural: una mirada descolonizadora, crítica e interseccional. En Villa A. y Martínez M. E. (Comps.). Relaciones escolares y diferencias culturales: la educaión en perspectiva intercultural (pp. 171-197). Ciudad Autónoma de Buenos Aires: Centro de Publicaciones Educativas y Material Didáctico.

Diez, M. L.; et al. (2011). Interculturalidad y educación. Cruces entre la investigación y la gestión. En Novaro, G. (Coord.). La interculturalidad en debate: experiencias formativas y procesos de identificación en niños indígenas y migrantes (pp. 265-281). Buenos Aires: Biblos.

Diez, M. L.; Thisted, S. y Martínez, M. E. (2015). Educación intercultural como política pública: el análisis de la experiencia de la provincia de Buenos Aires. Boletín de Antropología y Educación, 9, pp.163-168. Recuperado de http://antropologia.institutos.filo.uba.ar/sites/antropologia.institutos.filo.uba.ar/files/bae_n09_e03a03.pdf

Halbwachs, M. (2004). La memoria colectiva. Zaragoza: Prensas Universitarias de Zaragoza.

Hecht, A. C.; et al. (2015). Interculturalidad y educación en Argentina. Discusiones en torno a un concepto polisémico En Novaro, G.; Padawer, A. y Hecth, C. (Coords.). Educación, pueblos indígenas y migrantes: reflexiones desde México, Brasil, Bolivia, Argentina y España (pp.43-63). Buenos Aires: Biblos.

Hobsbawn, E. y Ranger, T. (Eds.). (2002). La invención de la tradición. Barcelona: Crítica.

Ingold, T. (2011). Being Alive: Essays on Movement, Knowledge, and Description. London: Routledge.

Jelin, E. (2018). La lucha por el pasado. Cómo construimos la memoria social. Ciudad Autónoma de Buenos Aires: Siglo $X X I$.

Levinson, B. A. y Holland, D. C. (1996). The cultural production of the educated person: An introduction. In Levinson, B. A., D.; Foley, E. y Holland, D. C. (Eds.). The cultural production of the educated person: critical ethnographies of schooling and local practice (pp. 1-56). Albany: State University of New York Press.

Lucas, M. (2018). Acciones educativas interculturales en la provincia de Buenos Aires (Argentina). Polifonías. Revista de Educación, 9, pp.81-106.

Nagy, M. A. (2013). Los museos de la última frontera bonaerense y sus narrativas acerca de los pueblos indígenas. Revista del Museo de Antropología, 6 (1), pp. 79-90. Recuperado de https://revistas.unc.edu.ar/index.php/antropologia/article/view/5506

Novaro, G. (2011). Niños y escuelas. Saberes en tensión. En Batallán, G. y Neufeld, M.R. (Coords.). Discusiones sobre infancia y adolescencia: niños y jóvenes dentro y fuera de la escuela (pp. 179-198). Buenos Aires: Biblos.

Padawer, A.; et al. (2017). Saber hacer. La participación en actividades de reproducción social, en las dimensiones expresivas de la vida social y en la escuela. Boletín de Antropología y Educación (8); 11, pp. 41-45. Recuperado de: http://hdl.handle.net/11336/82202

Padawer, A. (2013). El conocimiento práctico en poblaciones rurales del sudoeste misionero: habilidades y explicitaciones. Astrolabio; $10 \quad$ (7), pp.156-187. Recuperado de https://ri.conicet.gov.ar/bitstream/handle/11336/27456/CONICET_Digital_Nro.41f8c706-2652-423a-b27334e94c1745d6_A.pdf?sequence $=2 \&$ isAllowed $=y$

Pupio, A. (2013). El fortín Cuatreros en la conmemoración del pasado: ¿Qué historia(s) no han contado? En Pupio, A. y Perrière, H. Malones, fortines y estancias en la identidad de General Daniel Cerri (pp. 13-47). Bahía Blanca: EDIUNS

Ramos, A. (2011). Perspectivas antropológicas sobre la memoria en contextos de diversidad/desigualdad. Alteridades 21(42), pp. 131-148.

Ramos, A. (2016). La memoria como objeto de reflexión: recortando una definición en movimiento. En Ramos, A.; Crespo, C. y Tozzini, M. A. (Comps.) Memorias en lucha: recuerdos y silencios en contextos de subordinación y alteridad (pp. 51-69). Viedma: Editorial UNRN.

Rockwell, E. (2009). La experiencia etnográfica. Historia y cultura en los procesos educativos. Buenos Aires: Paidós.

Rosso, I. (2018). Buenos Aires indígena. Cartografía social de lo invisible. Tandil: Editorial UNICEN.

Thisted, S. (2014). Políticas, retóricas y prácticas educativas en torno a la cuestión de las "diferencias". Itinerarios de un siglo largo en el tratamiento de la cuestión de migrantes e indígenas. En Villa A.I. y Martínez, M. E. (Comps). Relaciones escolares y diferencias culturales: la educación en perspectiva intercultural (pp. 133-170).

Villa, A., et al. (2009). Procesos educativos y escolares en espacios interculturales. Decisio, pp.79-85. 
REVISTA DE LA ESCUELA DE CIENCIAS DE LA EdUCACIÓN, AÑo 17, NRO. 16, VOL. 1, ENERO A JUNIO DE 2021. PÁGINAS 58-67. ISSN 2362-3349 (EN LíNEA). MEMORIAS Y CONOCIMIENTOS INDÍGENAS: EXPERIENCIAS INTERCULTURALES Y EDUCACIÓN EN EL SUDOESTE DE LA PROVINCIA DE BUENOS AIRES. Hernán Perriére.

Otras Fuentes consultadas

Cámara de Diputados de la provincia de Buenos Aires (27 de junio de 2007). Ley de Educación Provincial. [Ley N. o 13688]. Recuperado de http://abc.gov.ar/primaria/sites/default/files/documentos/ley-13688-07.pdf

Dirección General de Cultura y Educación de la provincia de Buenos Aires. Resolución N.

3655. Marco General de la Política curricular. 2007. Recuperado de http://servicios.abc.gov.ar/lainstitucion/organismos/consejogeneral/disenioscurriculares/documentosdescarga/ marcogeneral.pdf

Ministerio de Educación, Ciencia y Tecnología (14 de diciembre de 2006). Ley de Educación

Nacional [Ley N. o 26206]. file:/// C:/Users/Lark/Downloads/1753-6521-1-PB\%20(1).pdf 